\title{
BMJ Open Liquorice-induced hypokalaemia in patients treated with Yokukansan preparations: identification of the risk factors in a retrospective cohort study
}

\author{
Saori Shimada, ${ }^{1}$ Tetsuaki Arai, ${ }^{2}$ Akira Tamaoka, ${ }^{3}$ Masato Homma ${ }^{1}$
}

To cite: Shimada S, Arai T, Tamaoka A, et al. Liquoriceinduced hypokalaemia in patients treated with Yokukansan preparations: identification of the risk factors in a retrospective cohort study. BMJ Open 2017;7:e014218. doi:10.1136/ bmjopen-2016-014218

- Prepublication history for this paper is available online. To view these files please visit the journal online (http://dx.doi org/10.1136/bmjopen-2016014218).

Received 9 September 2016 Revised 27 March 2017 Accepted 8 May 2017

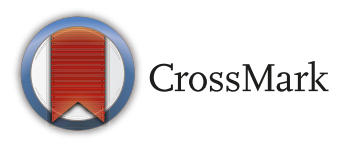

${ }^{1}$ Department of Pharmaceutical Sciences, Graduate School of Comprehensive Human Sciences, University of Tsukuba, Tsukuba, Japan

${ }^{2}$ Department of Psychiatry, Graduate School of

Comprehensive Human

Sciences, University of Tsukuba,

Tsukuba, Japan

${ }^{3}$ Department of Neurology,

Graduate School of

Comprehensive Human

Sciences, University of Tsukuba,

Tsukuba, Japan

Correspondence to

Dr Masato Homma;

masatoh@md.tsukuba.ac.jp

\section{ABSTRACT}

Objective To evaluate serum potassium levels and rates of hypokalaemia in patients treated with liquoricecontaining Japanese traditional Kampo-medicines Yokukansan (YK) and Yokukansan-ka-chinpihange (YKCH). Design Retrospective cohort study.

Setting Patients receiving YK preparations for dementia and other psychiatric disorders in the University of Tsukuba Hospital in Japan.

Participants 389 patients (male/female: 174/215, $68.6 \pm 16.1$ years) were treated with YK preparations for 231 days (range $6-2788$ days). Patients whose potassium levels were $<3.6 \mathrm{mEq} / \mathrm{L}$ before administration of $\mathrm{YK}$ preparations, and drug non-compliant patients, were excluded.

Main outcome measure The occurrence rate of hypokalaemia and assessment of the risk factors for YK preparation-induced hypokalaemia.

Results Of the 389 patients treated with YK preparations, $94(24.2 \%)$ developed hypokalaemia (potassium levels $<3.6 \mathrm{mEq} / \mathrm{L}$ ) 34 days (range 1-1600 days) after administration of the preparations. 36 (38.3\%) patients had co-administration with lower potassium-inducing drugs (LPIDs; diuretics, glucocorticoids, mineralocorticoids and glycyrrhizin), which was more frequent in the patients without hypokalaemia (17.3\%) $(p<0.05)$. A Cox proportional hazard model identified four risk factors for hypokalaemia: YK administration (not YKCH) (HR 3.093, $95 \% \mathrm{Cl} 1.408$ to 6.798 ), co-administration of LPIDs (HR $2.743,95 \% \mathrm{Cl} 1.754$ to 4.289 ), hypoalbuminaemia at baseline (HR 2.145, 95\% 1.360 to 3.384 ), and full dosage administration (7.5 g/day) (HR $1.600,95 \% \mathrm{Cl} 1.005$ to 2.549).

Conclusions Serum potassium monitoring should be done at least monthly in patients with the following risk factors: LPID co-administration, YK administration, hypoalbuminaemia, and full dosage administration.

\section{INTRODUCTION}

Yokukansan (YK) and Yokukansan-ka-chimpihange (YKCH) are Japanese kampo (traditional) medicines-consisting of seven and nine crude drug extracts, respectively (table 1)-for the treatment of restlessness and agitation in children. ${ }^{1}$ Current use of

\section{Strengths and limitations of this study}

- This is the first report to identify the risk factors for hypokalaemia as an initial symptom of pseudoaldosteronism in patients treated with YK preparations containing small amounts of glycyrrhiza ( $1.5 \mathrm{~g} /$ day).

- Patient data, including backgrounds and laboratory data, are under the sole stewardship of the University of Tsukuba Hospital.

- Since this is a retrospective cohort study, blood sampling intervals for assessing serum potassium and other laboratory data were not fixed.

YK preparations focuses on the treatment of behavioural and psychological symptoms of dementia (BPSD) in patients with Alzheimer's disease and Lewy body dementia. ${ }^{1-10}$ This trend has altered the YK target patient population from children to the elderly in just the past decade. ${ }^{11}$ An increase in adverse effects such as liver dysfunction, interstitial pneumonia, pseudoaldosteronism, and rhabdomyolysis have been found in dementia patients, leading to the revision of the YK preparation package insert. ${ }^{12}$ These adverse effects may be due to the change in target patient age (juvenile to elderly) and interactions with concomitant drugs being administered for the complications. ${ }^{11} 13$

Since both YK preparations contain liquorice as the crude drug glycyrrhiza, they have liquorice-induced pseudoaldosteronism characterised by hypertension and hypokalaemia as their essential adverse effect. ${ }^{14}$ This adverse effect has been ignored to date because the glycyrrhiza content of the preparation ( $1.5 \mathrm{~g}$ /day) is less than the $2.5 \mathrm{~g} /$ day which is considered to increase the risk of liquorice-induced pseudoaldosteronism among Kampo-medicines containing glycyrrhiza ${ }^{15}$ (table 2 ). However, several observations have revealed that the occurrence of 
Table 1 Components of YK preparations

\begin{tabular}{lll}
\hline & \multicolumn{2}{l}{ Weight (g/day) } \\
\cline { 2 - 3 } Constituent herbs & YK $^{*}$ & YKCH† \\
\hline JP Atractylodes Lancea Rhizome & 4.0 & 4.0 \\
\hline JP Poria Sclerotium & 4.0 & 4.0 \\
\hline JP Cnidium Rhizome & 3.0 & 3.0 \\
\hline JP Uncaria Hook & 3.0 & 3.0 \\
JP Japanese Angelica Root & 3.0 & 3.0 \\
JP Bupleurum Root & 2.0 & 2.0 \\
JP Glycyrrhiza & 1.5 & 1.5 \\
JP Pinellia Tuber & - & 5.0 \\
JP Citrus Unshiu Peel & - & 3.0 \\
\hline
\end{tabular}

${ }^{*} 7.5 \mathrm{~g}$ of Tsumura YK extract granules contains $3.25 \mathrm{~g}$ of a dried extract of the mixed crude drugs.

$\dagger 7.5 \mathrm{~g}$ of Tsumura YKCH extract granules contains $4.5 \mathrm{~g}$ of a dried extract of the mixed crude drugs.

JP, The Japanese Pharmacopoeia; YK, Yokukansan; YKCH, Yokukansan-ka-chimpihange.

hypokalaemia caused by YK preparations is unexpectedly high and may develop into life-threatening events such as congestive heart failure and rhabdomyolysis, which requires cessation of drug administration. ${ }^{16-18}$

In the present study, we retrospectively investigated the change in serum potassium levels in patients treated with YK preparations to assess the risk factor for hypokalaemia as an initial symptom of pseudoaldosteronism.

\section{METHODS}

\section{YK preparations}

YK preparations (YK and $\mathrm{YKCH}$ ) were obtained in a commercially available granule form (Tsumura \& Co, Tokyo, Japan), prepared from a mixture of seven and nine crude drug extracts, respectively (table 1 ). These

\begin{tabular}{|c|c|c|}
\hline $\begin{array}{l}\text { Glycyrrhiza } \\
\text { contents } \\
\text { (g/day) }\end{array}$ & $\begin{array}{l}\text { Number of } \\
\text { Kampo- } \\
\text { medicines }\end{array}$ & Examples \\
\hline 6.0 & 1 & Shakuyakukanzo-to \\
\hline 5.0 & 2 & $\begin{array}{l}\text { Kanbakutaiso-to, } \\
\text { Shakuyakukanzo-bushi-to }\end{array}$ \\
\hline 3.0 & 11 & $\begin{array}{l}\text { Ninjin-to, Oren-to, Shoseiryu-to, } \\
\text { etc }\end{array}$ \\
\hline 2.5 & 1 & Hangeshashin-to \\
\hline 2.0 & 38 & $\begin{array}{l}\text { Kakkon-to, Shosaiko-to, } \\
\text { Tokishigyaku-ka-goshuyushokyo- } \\
\text { to, etc }\end{array}$ \\
\hline 1.5 & 24 & $\begin{array}{l}\text { Hochuekki-to, Yokukansan, } \\
\text { Yokukansan-ka-chimpihange, etc }\end{array}$ \\
\hline 1.0 & 31 & $\begin{array}{l}\text { Chotosan, Ninjinyoei-to, } \\
\text { Rikkunshi-to, etc }\end{array}$ \\
\hline
\end{tabular}

traditional medicines are approved for medical use in Japan. The daily dose of both YK preparations is $7.5 \mathrm{~g} /$ day as the granule, which contains a dried extract of the mixed crude drugs of YK (3.25 g) or YKCH (4.5 g) as shown in table 1 . The glycyrrhiza content for both YK and $\mathrm{YKCH}$ was the same as the daily dose extracts $(1.5 \mathrm{~g})$ (tables 1 and 2).

\section{Patients and study design}

Three hundred and eighty-nine patients (174 males and 215 females, mean \pm SD age $68.6 \pm 16.1$ years) receiving YK preparations for BPSD or other psychiatric disorders were enrolled at the University of Tsukuba Hospital from March 2007 to January 2016 (table 3). One hundred and eighty-four patients were treated as outpatients and 205 were admitted during the trial. Three hundred and twenty-three patients were treated with YK and 66 with YKCH for 231 days (range 6-2788 days). YK preparations were given orally before or after meals at full dose strength (2.5 g three times a day; $7.5 \mathrm{~g} /$ day) or a reduced dose (2.5 $\mathrm{g}$ once or twice a day; $2.5-5.0 \mathrm{~g} /$ day) based on patient symptoms; 229 patients $(58.9 \%)$ received a full dose of YK preparation. Non-compliant patients as well as those whose pre-administration serum potassium level was $<3.6$ $\mathrm{mEq} / \mathrm{L}$ were excluded from the study. Changes in laboratory data, including serum potassium, sodium, chloride, aspartate aminotransferase, alanine aminotransferase, blood urea nitrogen, serum creatinine and albumin, and co-medication were retrospectively investigated via medical records before and after administration of YK preparations.

\section{Statistical analyses}

Statistical parameters were ascertained using SPSS software (International Business Machines Corp, Armonk, New York, USA). Statistical analyses were performed by the Mann-Whitney test and $\chi^{2}$ test for comparing differences between hypokalaemic and non-hypokalaemic groups. The cut-off threshold for hypokalaemia was determined by a receiver operating characteristic (ROC) curve. Intergroup differences in patients treated with and without lower potassium-inducing drugs (LPIDs; diuretics, glucocorticoids, mineralocorticoids and glycyrrhizin preparations) were analysed by the Kaplan-Meier method. A Cox proportional hazard model was employed to identify risk factors for hypokalaemia. A value of $\mathrm{p}<0.05$ was considered to be statistically significant.

\section{RESULTS}

Ninety-four patients $(24.2 \%)$ developed hypokalaemia (potassium levels $<3.6 \mathrm{mEq} / \mathrm{L}$ ) during the study period (table 3). The median time to develop hypokalaemia was 34 days (range 1-1600 days) after administration of YK preparations (table 3) at which the cumulative rate of hypokalaemia was $12.7 \%$ by Kaplan-Meier analysis (figure 1).

Significant differences between hypokalaemic and non-hypokalaemic patients were observed and attributed 
to the type of drug used (YK or YKCH), the dosed amount, and dosing period. Compared with the non-hypokalaemic group, the hypokalaemic group received YK more often than YKCH $(91.5 \%$ vs $80.3 \%)$ as well as more full dosing amounts $(70.2 \%$ vs $55.3 \%, \mathrm{p}<0.05)$ (table 3 ). The dosing period in hypokalaemic cases was significantly shorter than in non-hypokalaemic cases (169 days, range 8-2280 days vs 266 days, range 6-2788 days, $\mathrm{p}<0.05$ ) (table 3 ).

Of the patients with hypokalaemia, $36(38.3 \%)$ were receiving concomitant doses of LPIDs, which was more frequent in the patients without hypokalaemia (17.3\%) $(\mathrm{p}<0.05)$ (table 3). Mean serum potassium at baseline in the hypokalaemic group was also lower than in the non-hypokalaemic group $(\mathrm{p}<0.001)$, even though their values were within the normal range. The reduction for serum potassium ( $\Delta$ potassium) after administration of YK preparations was pronounced in the hypokalaemic group compared with the non-hypokalaemic group $(-0.7,-3.0$ to $-0.1 \mathrm{mEq} / \mathrm{L}$ vs $-0.1,-1.3$ to $1.1 \mathrm{mEq} / \mathrm{L}, \mathrm{p}<0.001$ ) (table 3 ).

Hypokalaemic patients more often displayed abnormal values of alanine aminotransferase, albumin and blood urea nitrogen at baseline than the non-hypokalaemic group $(14.9 \%$ vs $9.8 \%, 50.0 \%$ vs $29.2 \%$ and $39.4 \%$ vs $26.4 \%$, respectively, $\mathrm{p}<0.05)$. A higher rate of hypoalbuminaemia (albumin $<3.8 \mathrm{~g} / \mathrm{L}$ ) was also observed in hypokalaemic patients $(45.7 \%$ vs $28.8 \%, \mathrm{p}<0.05)$, with concomitant minimum potassium levels during the administration of YK preparations (data not shown).

A Cox proportional hazard model based on univariable and multivariable analysis identified four risk factors for YK preparation-induced hypokalaemia: YK administration (not YKCH) (HR 3.093, 95\% CI 1.408 to 6.798),

Table 3 Demographic data of the subjects

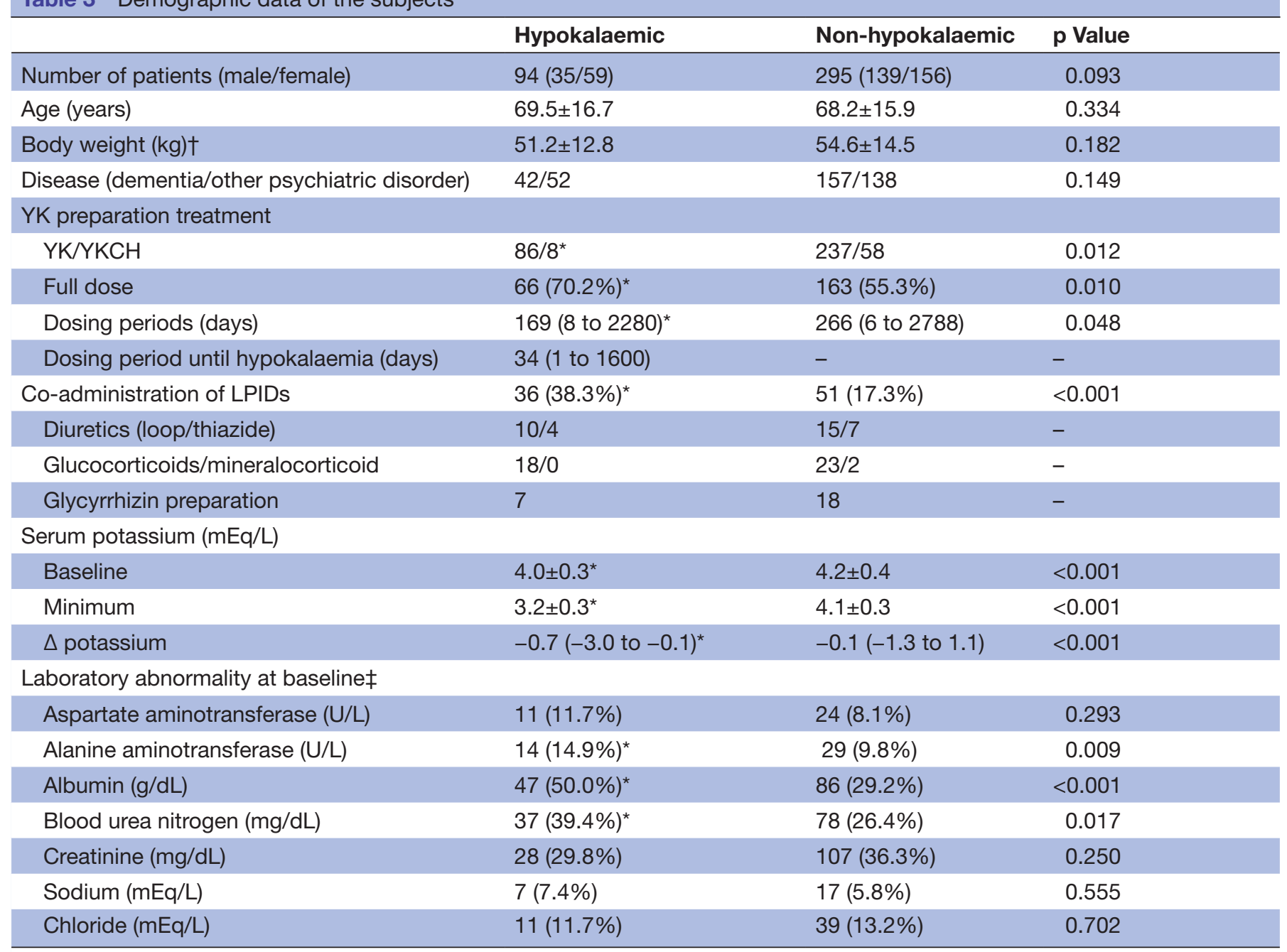

The data are presented as number of patients, mean \pm SD or median (range).

${ }^{*} \mathrm{p}<0.05$ versus non-hypokalaemia.

†The number of patients whose weight was recorded was 85 with hypokalaemia and 224 with non-hypokalaemia.

$\ddagger$ The number of patients with abnormal laboratory data at baseline.

The normal ranges for laboratory data are: aspartate aminotransferase, 8.0-38.0 U/L; alanine aminotransferase, 4.0-44.0 U/L; albumin, 3.8-5.3 g/dL; blood urea nitrogen, 8.0-20.0 mg/dL; creatinine, 0.61-1.04 mg/dL (male) and 0.47-0.79 mg/dL (female); sodium, $135.0-147.0$ $\mathrm{mEq} / \mathrm{L}$; chloride, $98.0-108.0 \mathrm{mEq} / \mathrm{L}$.

LPIDs, lower potassium-inducing drugs; YK, Yokukansan; YKCH, Yokukansan-ka-chimpihange. 


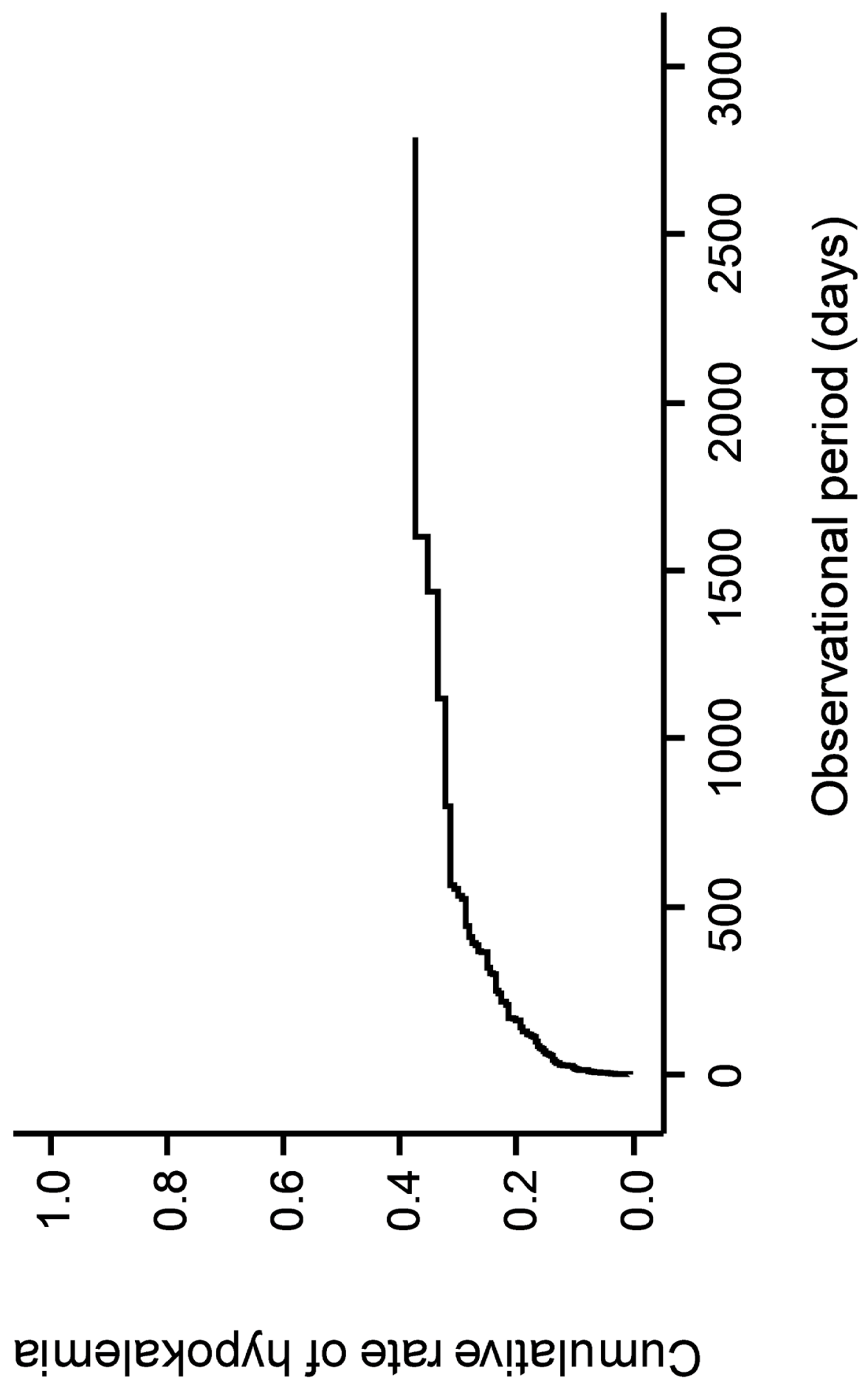


Table 4 Hazard ratios for hypokalaemia in patients treated with YK preparations ${ }^{\star}$

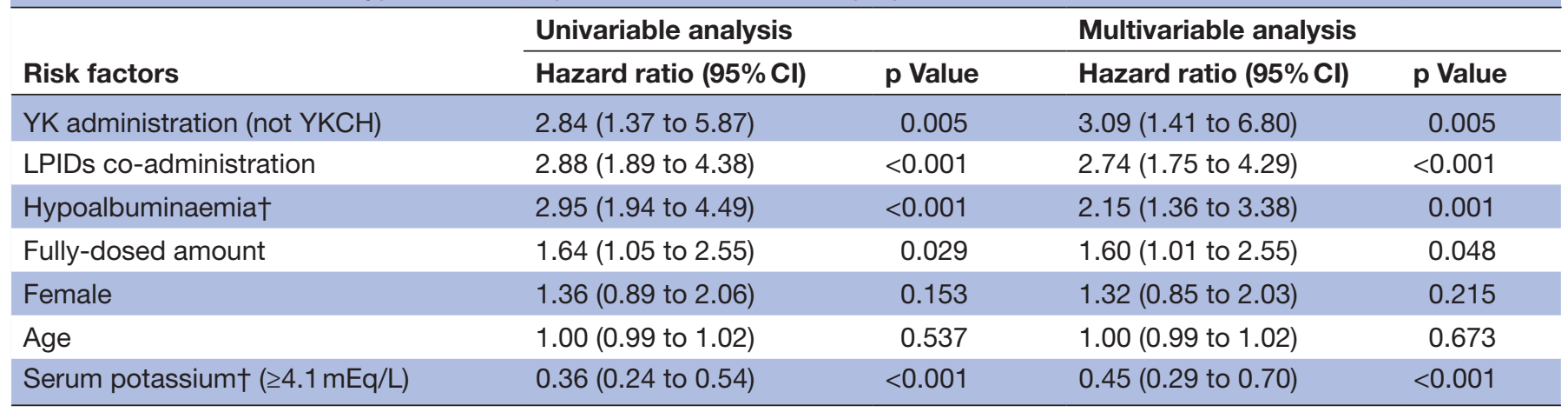

${ }^{*}$ Cox proportional hazard model was used.

†Determined at baseline.

LPIDs, lower potassium-inducing drugs; YK, Yokukansan; YKCH, Yokukansan-ka-chimpihange.

co-administration of LPIDs (HR 2.743, 95\% CI 1.754 to 4.289), hypoalbuminaemia at baseline (HR 2.145, 95\% CI 1.360 to 3.384 ), and full-dose administration of YK preparations ( $7.5 \mathrm{~g} /$ day) (HR $1.600,95 \% \mathrm{CI} 1.005$ to 2.549 ) (table 4). On the other hand, baseline potassium levels $\geq 4.1 \mathrm{mEq} / \mathrm{L}$, established as the optimal threshold to predict non-hypokalaemia, were a reverse risk factor for hypokalaemia (HR $0.450,95 \%$ CI 0.288 to 0.702 ) (table 4 ).

To assess the effects of LPIDs co-administration on the occurrence of hypokalaemia, the time between administration of YK preparations and development of hypokalaemia was compared between groups with and without LPIDs (figure 2). Patients treated with concomitant LPIDs showed a shorter time-to-occurrence for hypokalaemia than those without concomitant LIPDs $(\mathrm{p}<0.001)$ (figure 2$)$.

Nine patients discontinued YK preparations due to hypokalaemia and each had the risk factors indicated in table 5. Seven patients (except for cases 4 and 7) had multiple risk factors. Cases 1 and 2 developed severe hypokalaemia, with potassium levels $<2.1 \mathrm{mEq} / \mathrm{L}$, and were found to have been co-administered a thiazide diuretic or presented with rhabdomyolysis, respectively (table 5).

\section{DISCUSSION \\ Occurrence rate of hypokalaemia when using YK preparations}

Both YK preparations contain $1.5 \mathrm{~g}$ /day of glycyrrhiza (table 1), which is much less than the Shakuyakukanzo-to $(6.0 \mathrm{~g} /$ day of glycyrrhiza) preparation that is thought to possess the highest risk for pseudoaldosteronism among the Kampo-medicines (table 2). ${ }^{19-21}$ However, the Japanese Adverse Drug Event Report (JADER), a spontaneous adverse events reporting system, currently reports that YK-induced pseudoaldosteronism rates are comparable with those of Shakuyakukanzo-to, even though the possible risk should be low in terms of the glycyrrhiza content. ${ }^{1920}$ Present results seemed to confirm the JADER's report; hypokalaemia was found in high frequency, with $24.2 \%$ of the patients having been treated by YK preparations. This rate is comparable with a previous investigation in elderly patients, where $17 \%$ of patients treated with YK developed hypokalaemia. ${ }^{22}$

On the other hand, an adverse drug reaction (ADR) frequency investigation on YK for ethical use reported that hypokalaemia occurred in $1.3 \%$ of patients treated with YK, ${ }^{13}$ which was considerably lower than our observation. Several factors may explain this difference in the occurrence rate of hypokalaemia. One possible reason is patient background in terms of disease severity, complications and concomitant drug administration. Eighty per cent of the subjects in the ADR investigation were outpatients and $61.9 \%$ of the patients had no complications and no medication for dementia. ${ }^{13}$ On the other hand, this study enrolled patients who presented with complicating psychiatric disorders $(48.8 \%)$ and received various medications, including LPIDs such as diuretics, glucocorticoids, mineralocorticoids and glycyrrhizin preparations. Another possible reason is the observation periods between the studies. The ADR frequency investigation did not track adverse events longer than 52 weeks after starting YK administration. ${ }^{13}$

\section{Serum potassium monitoring to prevent YK preparation- induced hypokalaemia}

Liquorice-induced pseudoaldosteronism due to Kampo-medicines can escalate into a serious event that makes hospitalisation necessary. The mechanism seems to be clear. ${ }^{23-26}$ Glycyrrhetinic acid (GA), a metabolite of glycyrrhizin (GL) contained in liquorice, has been found to be the major substance responsible for pseudoaldosteronism. GA inhibits $11 \beta$-hydroxysteroid dehydrogenase type 2 (11 $\beta$-HSD 2$)$, which catalyses the conversion of cortisol to cortisone and prevents the binding of cortisol to the mineralocorticoid receptor (MCR) in the mineralocorticoid target tissues. This inhibition leads to increased cortisol levels in the tissues and excess cortisol binding to the MCR with the same affinity as aldosterone. ${ }^{23} 24$ The MCR activation increases sodium reuptake and inhibits potassium reabsorption in the kidney, resulting in hypertension, metabolic alkalosis and hypokalaemia. ${ }^{25}$ Monitoring of serum potassium levels, therefore, is useful for the early detection and assessment of the severity of 


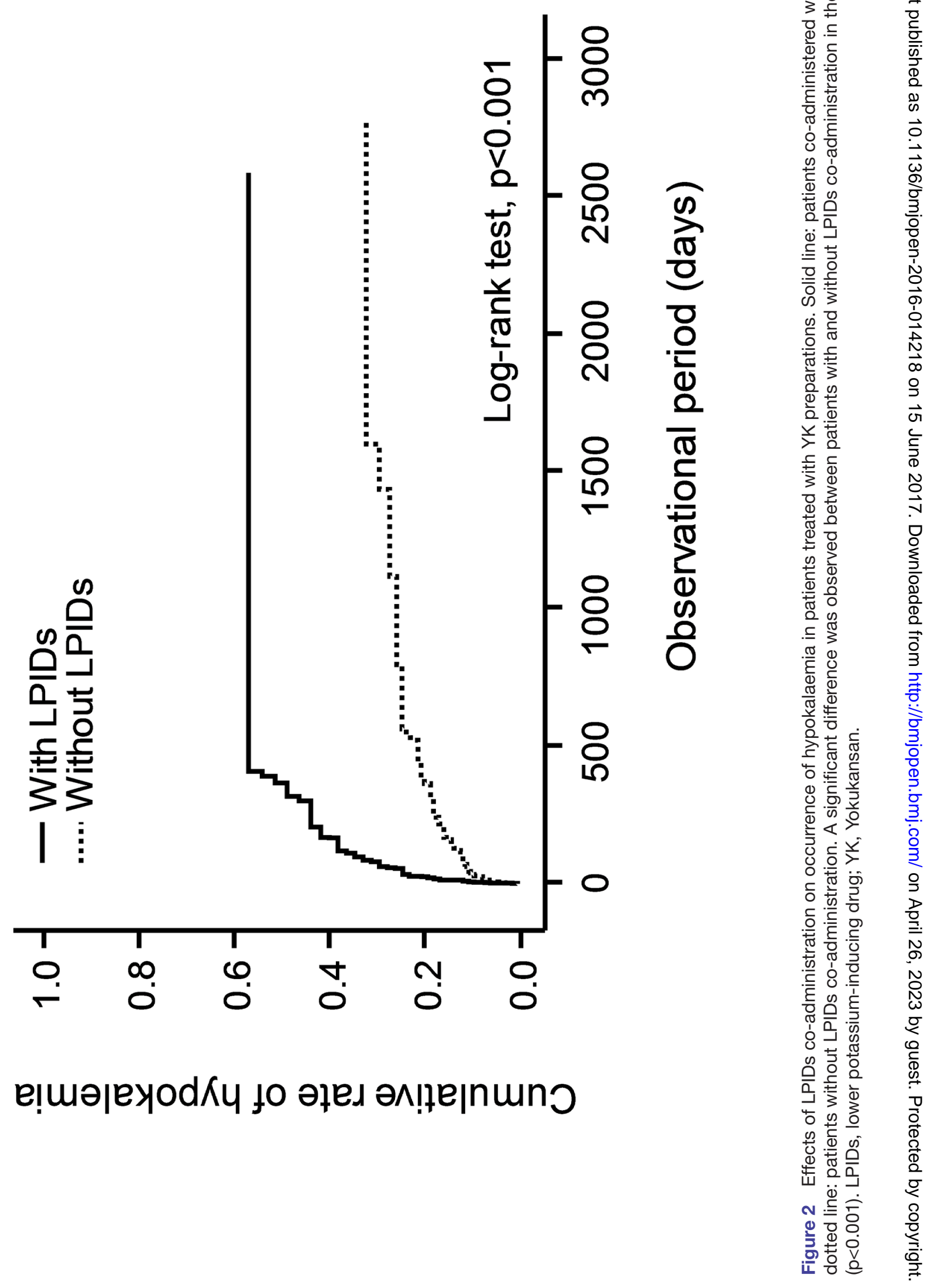




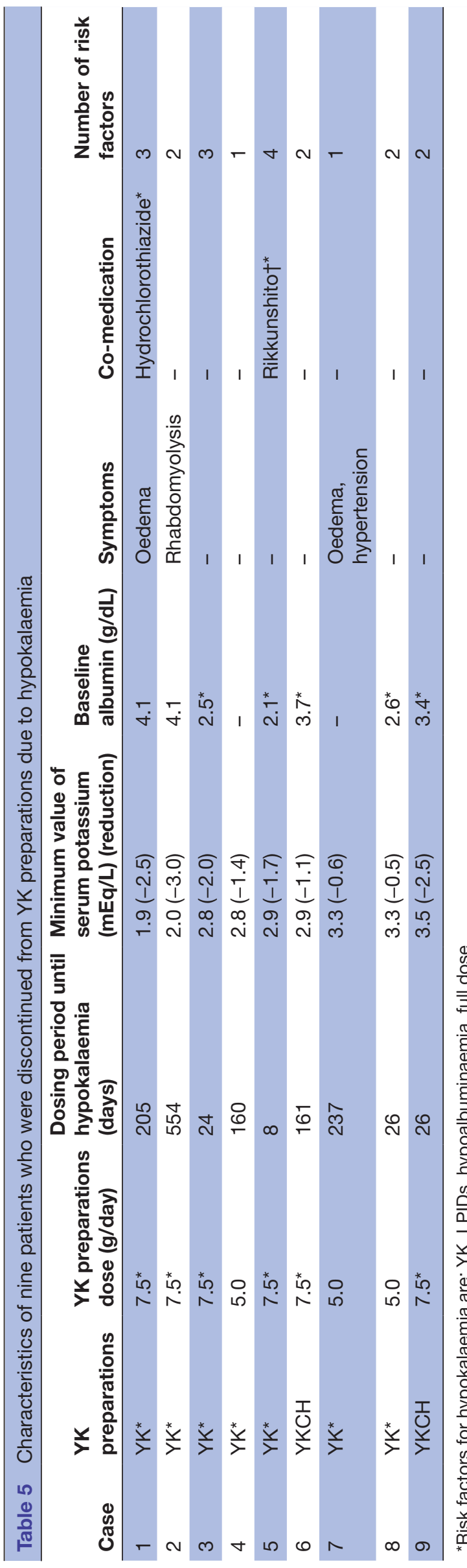

pseudoaldosteronism. Our present results suggest that serum potassium levels should be checked the first month after starting YK preparations, because the median time for hypokalaemia onset was 34 days after administration (table 3). Serum potassium monitoring should be continued during treatment, because late onset pseudoaldosteronism was found to occur up to 3 years after final administration of Kampo-medicines containing glycyrrhiza. ${ }^{27}$

\section{Risk factors for YK preparation-induced hypokalaemia}

We found four risk factors associated with hypokalaemia in patients with heterogeneous clinical backgrounds (table 4). The risk of hypokalaemia during YK treatment was 3.09 times higher than that of YKCH (table 4). One possible explanation of this finding may be due to the difference in GL content between $\mathrm{YK}$ and $\mathrm{YKCH}$, even though the glycyrrhiza content is the same $(1.5 \mathrm{~g} /$ day $)$ as shown in table 1. Kampo-medicines, including YK preparations, are spray-dried herbal extracts (table 1). A mixture of seven or nine crude drugs are added to water and boiled, filtered, concentrated and then the resulting decoctions are further spray-dried to yield the extract for making YK preparations. In this manufacturing process, the wet extraction rate of GL may differ between YK and YKCH due to variations in the combination of crude drugs and the $\mathrm{pH}$ value of their decoctions. ${ }^{28}$ A resultant higher GL content for YK might therefore present a higher risk of hypokalaemia compared with YKCH.

Patients co-administered with LPIDs were 2.74 times more likely to develop hypokalaemia (table 4, figure 2) and experience a shorter time-to-onset compared with YK preparations alone (figure 2). Among the LPIDs, loop and thiazide diuretics attract special attention because they are frequently prescribed for dementia patients with hypertension (data not shown). Severe hypokalaemic cases with low potassium levels of $1.9 \mathrm{mEq} / \mathrm{L}$ had received thiazide diuretics concomitantly (table 5 ).

Patients with hypoalbuminaemia had a 2.15 times higher rate of hypokalaemia (table 4). Since $99.9 \%$ of circulating GA are bound to albumin, ${ }^{29}$ hypoalbuminaemia may increase the unbound fraction of GA through pharmacokinetic alteration, resulting in an enhancement of the pharmacological actions of GA. Yoshino and colleagues have suggested that hypoalbuminaemia was a risk factor for pseudoaldosteronism in three other Kampo-medicines. ${ }^{30}$ The present results are the first to report that hypoalbuminaemia is a possible risk factor for liquorice-induced hypokalaemia due to YK preparations.

The occurrence of hypokalaemia might be dose dependent in patients treated with YK preparations because full-dose YK preparations $(7.5 \mathrm{~g} /$ day $)$ increased the risk more than 1.60 times compared with a reduced dose (table 4). This observation is consistent with previous reports suggesting that liquorice-induced pseudoaldosteronism was found in a dose-dependent manner. ${ }^{2021}$ Since a majority of the dementia patients taking YK preparations were elderly, the reduced dose is recommended 
for any patients with risk factors for pseudoaldosteronism. Although age was not identified as a risk factor for hypokalaemia in the present study, this might be due to a lack of comparison, as most of the patients investigated were elderly (mean age $68.6 \pm 16.1$ years old). Initiation of full-dose YK preparations should therefore be avoided in elderly patients whose $11 \beta-H S D$ activity might be low due to an age-dependent decline in kidney function. ${ }^{31}$ In fact, seven of nine patients who discontinued YK preparations due to hypokalaemia were over 70 years old and had multiple risk factors for hypokalaemia (table 5).

\section{CONCLUSION}

Hypokalaemia was found at an unexpectedly high rate in patients receiving treatment with YK preparations, even though the liquorice content was relatively small. Four risk factors were found to be important in elderly patients receiving long-term treatment with YK preparations: YK versus YKCH administration, LPIDs co-administration, hypoalbuminaemia, and full dosage administration $(7.5 \mathrm{~g} /$ day $)$. It is recommended that serum potassium monitoring should be done at least monthly for the safe use of YK preparations in patients with multiple risk factors.

Correction notice This paper has been amended since it was published Online First. Owing to a scripting error, some of the publisher names in the references were replaced with 'BMJ Publishing Group'. This only affected the full text version, not the PDF. We have since corrected these errors and the correct publishers have been inserted into the references.

Acknowledgements We gratefully acknowledge Drs K Doki, T Hosono and $Y$ Suzuki for their useful advice and discussions. We would also like to thank Dr Bryan $\mathrm{J}$ Mathis of the Medical English Communication Centre (University of Tsukuba) for proofreading this article.

Contributors SS, TA, AT and MH designed and supervised the study. TA and AT selected the patients for this study. SS and MH corrected the data and carried out statistical analyses. SS and MH drafted the original manuscript and all authors checked and revised the manuscript. SS and MH are the guarantors.

\section{Competing interests None declared.}

Patient consent This is an observational study that does not require written consent to be obtained from each patient. The study design was described on a web page which patients can access from our hospital home page. If the patients do not want to attend the study, they can inform us of their intentions.

Ethics approval The Ethical Committee of the University of Tsukuba Hospital.

Provenance and peer review Not commissioned; externally peer reviewed.

Data sharing statement The full dataset is available from the corresponding author.

Open Access This is an Open Access article distributed in accordance with the Creative Commons Attribution Non Commercial (CC BY-NC 4.0) license, which permits others to distribute, remix, adapt, build upon this work non-commercially, and license their derivative works on different terms, provided the original work is properly cited and the use is non-commercial. See: http://creativecommons.org/ licenses/by-nc/4.0/

(C) Article author(s) (or their employer(s) unless otherwise stated in the text of the article) 2017. All rights reserved. No commercial use is permitted unless otherwise expressly granted.

\section{REFERENCES}

1. Iwasaki K, Satoh-Nakagawa T, Maruyama M, et al. A randomized, observer-blind, controlled trial of the traditional Chinese medicine YiGan San for improvement of behavioral and psychological symptoms and activities of daily living in dementia patients. J Clin Psychiatry 2005;66:248-52.

2. Mizukami K, Asada T, Kinoshita T, et al. A randomized cross-over study of a traditional Japanese medicine (kampo), yokukansan, in the treatment of the behavioural and psychological symptoms of dementia. Int J Neuropsychopharmacol 2009;12:191-9.

3. Shinno H, Inami Y, Inagaki T, et al. Effect of Yi-Gan San on psychiatric symptoms and sleep structure at patients with behavioral and psychological symptoms of dementia. Prog Neuropsychopharmacol Biol Psychiatry 2008;32:881-5.

4. Monji A, Takita M, Samejima T, et al. Effect of yokukansan on the behavioral and psychological symptoms of dementia in elderly patients with Alzheimer's disease. Prog Neuropsychopharmacol Biol Psychiatry 2009;33:308-11.

5. Okahara $\mathrm{K}$, Ishida $\mathrm{Y}$, Hayashi $\mathrm{Y}$, et al. Effects of yokukansan on behavioral and psychological symptoms of dementia in regular treatment for Alzheimer's disease. Prog Neuropsychopharmacol Biol Psychiatry 2010;34:532-6.

6. Hayashi $Y$, Ishida $Y$, Inoue T, et al. Treatment of behavioral and psychological symptoms of Alzheimer-type dementia with yokukansan in clinical practice. Prog Neuropsychopharmacol Biol Psychiatry 2010;34:541-5.

7. Iwasaki $\mathrm{K}$, Kosaka $\mathrm{K}$, Mori $\mathrm{H}$, et al. Improvement in delusions and hallucinations in patients with dementia with Lewy bodies upon administration of yokukansan, a traditional Japanese medicine. Psychogeriatrics 2012;12:235-41.

8. Sumiyoshi H, Mantani A, Nishiyama S, et al. Yokukansan treatment of chronic renal failure patients receiving hemodialysis, with behavioral and psychological symptoms of dementia: an open-label study. $A m \mathrm{~J}$ Geriatr Psychiatry 2013;21:1082-5.

9. Miyazawa J. Psychiatric pharmacology: study of the clinical efficacy of yokukansankachimpihange on Alzheimer's disease. Psychiatry 2009;14:535-42.

10. Magome A. Effect of yokukansankachimpihange on dementia including the point of view of oriental medicine. Psychiatry 2011;18:108-14.

11. Homma M. [Education program of kampo-medicine for undergraduates in preparation for clinical setting]. Yakugaku Zasshi 2016;136:417-22.

12. Information of the package insert of $Y K$ preparations, Tsumura Kampo Square [Internet]. Tokyo: Tsumura \& CO. Tsumura Kampo Square; 2009-2016. http://www.tsumura.co.jp/password/m_square/ products/ichiran/index.htm. (accessed Jul 7 2016).

13. Hisada T, Maki A, Katori $Y$, et al. Adverse drug reaction frequency investigation of TSUMURA yokukansan extract granules for ethical use. Diagnosis Treat 2014;102:1577-89.

14. Conn JW, Rovner DR, Cohen EL, et al. Licorice-Induced pseudoaldosteronism; Hypertension, hypokalemia, aldosteronopenia, and suppressed plasma renin activity. JAMA 1968;205:492-6.

15. Issued by the Pharmaceutical and Supply Bureau, Ministry of Health and Welfare [Internet]. Tokyo: Japanese Ministry of Health and Welfare, 1978. http://www.japal.org/contents/19780213_158.pdf. (cited Jul 7 2016).

16. Nishiyama N, Takeshita M, Tanaka K, et al. [A case of severe hypokalemia caused by a Chinese herbal remedy (Yokukansan) in an 81-year-old woman with dementia]. Nihon Ronen Igakkai Zasshi 2011;48:553-7.

17. Yamada S, Tokumoto M, Kansui Y, et al. Severe metabolic alkalosis, hypokalemia, and respiratory acidosis induced by the Chinese herbal medicine yokukansan in an elderly patient with muscle weakness and drowsiness. CEN Case Rep 2013;2:23-7.

18. Kamei S, Kaneto $\mathrm{H}$, Irie $\mathrm{S}$, et al. Pseudoaldosteronism induced by yokukansan in an elderly Japanese type 2 diabetic patient with Alzheimer's disease. J Diabetes Investig 2015;6:487-8.

19. Shimodaira $\mathrm{H}$, Nozaki M, Kwon $\mathrm{Y}$, et al. Analysis of adverse reaction in kampo-medicines using JADER database of PMDA. lyakuhin Johogaku 2014;16:16-22.

20. Kato Y, Umetsu R, Hosoya N, et al. Analysis of licorice-induced pseudoaldosteronism in the Japanese adverse drug event report database. Traditional \& Kampo Medicine 2016;3:63-70

21. Homma M, Ishihara M, Qian W, et al. [Effects of long term administration of Shakuyaku-kanzo-To and Shosaiko-To on serum potassium levels]. Yakugaku Zasshi 2006;126:973-8.

22. Kumakiri C, Murakami A. A clinical effect of Yi-Gan San in elderly patients. Jounal Kampo Med 2008;55:1311-23. 
23. Stewart P, Valentino R, Wallace AM, et al. Mineralocorticoid activity of liquorice: 11-beta-hydroxysteroid dehydrogenase deficiency comes of age. The Lancet 1987;330:821-4.

24. Funder J, Pearce P, Smith R, et al. Mineralocorticoid action: target tissue specificity is enzyme, not receptor, mediated. Science 1988;242:583-5.

25. Farese RV, Biglieri EG, Shackleton $\mathrm{CH}$, et al. Licorice-induced hypermineralocorticoidism. N Engl J Med 1991;325:1223-7.

26. Xu R, Liu X, Yang J. A semi-physiologically based pharmacokinetic pharmacodynamic model for glycyrrhizin-induced pseudoaldosteronism and prediction of the dose limit causing hypokalemia in a virtual elderly population. PLoS One 2014;9:e114049.

27. Mantani N, Oka H, Watanabe T, et al. A case of pseudoaldosteronism that occurred in 63-year-old woman for the first time after 3-year administration of tokishigyakukagoshuyushokyoto. Kampo Medicine 2016;67:72-4.

28. Tada M, Kojima R, Nagata K, et al. The Pharmaceutical Society of Japan. 136th annual meeting of the pharmaceutical society of Japan 2016 Mar 26-29. Yokohama, Japan.

29. Ishida S, Ichikawa T, Sakiya Y. Binding of glycyrrhetinic acid to rat plasma, rat serum albumin, human serum, and human serum albumin. Chem Pharm Bull 1988;36:440-3.

30. Yoshino T, Nakamura H, Sano M, et al. Elevated direct bilirubin: possible predictors for pseudoaldosteronism: case-control study. Traditional \& Kampo Medicine 2016;3:174-6.

31. Homma M, Tanaka A, Hino K, et al. Assessing systemic 11[beta ]-hydroxysteroid dehydrogenase with serum cortisone/cortisol ratios in healthy subjects and patients with diabetes mellitus and chronic renal failure. Metabolism 2001;50:801-4. 